\title{
Adaptive Collocation Method for Simultaneous \\ Heat and Mass Diffusion with Phase Change
}

\author{
by
}

\author{
T. C. Chawla ${ }^{\dagger}$, D. R. Pedersen ${ }^{\dagger}$, G. Leaf ${ }^{\dagger}$, \\ W. J. Minkowycz*, and $A, R$. Shouman ${ }^{\star \star}$ \\ Reactor Analysis and Safety Division \\ Argonne National Laboratory \\ Argonne, IL 60439
}

\section{DISCLAIMER}

\begin{abstract}
This report was prepared as an account of work sponsored by an agency of the United States Government. Neither the United States Government nor any agency thereof, nor any of their employees, makes any warranty, express or implied, or assumes any legal liability or responsibility for the accuracy, completeness, or usefulness of any information, apparatus, product, or process disclosed, or represents that its use would not infringe privately owned rights. Reference herein to any specific commercial product, process, or service by trade name, trademark, manufacturer, or otherwise dnes not necessarily constitute or imply its endorsement, recommendation, or favoring by the United States Government or any agency thereof. The views and opinions of authors expressed herein do not necessarily state or reflect those of the United States Government or any agency thereof.
\end{abstract}

\footnotetext{
This work was performed under the auspices of the U. S. Department of Energy.

*Department of Mechanical Engineering University of Illinois at Chicago Circle Post office Box 4348

Chicago, IL 60680
**Department of Mechanical Engineering New Mexico State University
Las Cruces, NM 88003




\section{ABSTRACT}

In post-accident heat removal applications the use of a lead slab is jeing considered for protecting a porous bed of steel shot in ex-vessel cavity from direct impingement of molten steel or fuel as released from reactor vessel following a hypothetical core disassembly accident in an LMFBR. The porous bed is provided to increase the coolability of the fuel debris by the sodium coolant. The present study is carried out to determine melting rates of lead slab of various thicknesses by contact with sodium coolant and to evaluate the extent of penetration and the mixing rates of molten lead into liquid sodium by molecular diffusion alone. The study shows that these two calculations cannot be performed simultaneous without the use of adaptive coordinates which cause considerable stretching of the physical coordinates for mass diffusion. Because of the large difference in densities of these two liquid metals, the traditional constant density approximation for the calculation of mass diffusion cannot be used for studying their interdiffusion. The use of orthogonal collocation method along with adaptive coordinates produces extremely accurate results which are ascertained by comparing with the existing analytical solutions for concentration distribution for the case of constant density approximation and for melting rates for the case of infinite lead slab. The analysis further shows that the melting rate progressively increases as the thickness of lead slab decreases. The mixing of two liquid metals by molecular diffusion alone is extremely slow and the molten lead is likely to stay separated from sodium coolant unless fret convection mechanisms exist in the sodium. Before any significant mixing takes place the lead upon melting will sink to the bottom of porous bed and not participate in removing heat from fuel deuris lying on the sop of the porous steel bed. 
INTRODUCTION

In post-accident heat removal (PAHR) considerations following a failure of pressure vessel in a hypothetical LMFBR core disassembly accident, the release of molten core debris into ex-vessel cavity is of major concern. To protect the ex-vessei cavity floor from failure numerous designs are under consideration. In one such consideration, a slab of lead in the range of 10-20 cms in thickness is placed over a porous bed of stainless shots (provided for improved heat transfer by sodium coolant) lying over the exvessel cavity floor. The slab of lead is expected to protect the porous bed of stainless shots against direct impingement of molten steel or fuel as released from the pressure vessel. Since the ex-vesse? cavity is considerably larger in diameter than the pressure vessel, it is expected that sodium coolant will be in direct contact with the lead slab in the outer (relative to pressure vessel) part of the ex-vessel cavity. It is therefore of interest to determine: (1) the rate at which lead will melt upon contact with the pool of sodium and (2) the rate and the extent of penetration by molecular diffusion of molten lead into sodium. Molten lead is completely miscible in liquid sodium; however, because of large density differences, initially the only mechanism available for mixing of molten lead into sodium coolant is molecular diffusion or possibly by natural convection in the sodium pool in the presence of heat sources such as due to a layer of fuel debris lying on the lead slab. The effect of natural convection in the sodium pool is outside the subject matter of the present study. In the present study we will merely evaluate the rate and the extent of mixing molten lead into sodium by molecular diffusion.

The study of simultaneous molecular diffusion of heat and mass in a liquid-liquid system especially with large density differences such as between 
molten lead and sudium presents a considerable challenge in view of the foliowing two considerations: (1) the rate of molecular mass diffusion is considerably slower than that for heat diffusion and therefore the extent of the penetration of mass diffusion at any given time is sianificantly smaller than that for heat diffusion, and (2) owing to large density difference between sodium and lead, constant mixture density approximation for mass diffusion is not valid and therefore an analytical solution though available for constant density approximation is not applicable to lead-sodiun diffusion. As will be demonstrated, a constant density approximation leads to a significant error in the solution of mass diffusion probiem for the system under consideration. Consequently, a solution of the problem has to be pursued numerically. However, because of the above considerations, a solution by numerical methods presents a consideratie challenge. The encrmous difference in the diffusion rates for hear and mass introduces stiffness in the system of ODEs resulting from spatial discretization of the PDEs governing these two diffusion processes. Therefore finite differencing with respect to time requires special handling. Significant difference in penetration distances of heat and mass diffusion results in an extremely pour spatial resolution for mass diffusion if the same mesh distribution is used in physical coordinates for calculating simultaneously both of these processes. Since the width of the field for mass diffusion is considerably smaller than that for the heat diffusion, the mesh distribution will correspondingly have to be very closely spaced in the field where computations of rass diffusion are required. With the conventional methods, the number and the density of meshes required for the calculation of simultaneous heat and mass diffusion greatly exceed those that are theoretically necessary for normal engineering accuracy. To circumvent these difficulties we have devised 
special coordinate system to span the field in which mass diffusion is important. Coupled with this coordinate system we will utilize a collocation method with Hermite splines as approximating functions and Gaussian quadrature points as the collocation points. This method is known (see for example Refs. [1] and [2]) for very high accuracy both for moving boundary problems and problems that contain very sharp variation of dependent variables or shock fronts.

FORMLATIION OF MODEL AND GOVERNING EQUATIONS

Consider a slab of lead of finite thickness $L$ at room temperature brought suddenty into contact with hot liquid sodium pool of very large height. The initial temperature of sodium is such that upon contact, lead begins to melt immediately. The molten lead which is completely miscible in all proportions in sodium liquid, begins to diffuse into sodium and sodium into molten lead. However, the mass diffusion process is considerably slower than the melting rate of lead, therefore, at all times ample moltan lead is available for diffusion. The lower side of lead is in contact with a porous bed with porosity exceeding $50 \%$ and is filled with gases. For simplicity in modeling the lower side of the slab has been assumed to have negligible cooling. Both lead and sodium extend sufficientiy far enough laterally such that heat and mass transfer can be assumed to be one-dimensional through the thicknesses of these materials. Figure 1 shows schematically the geometry of the above system. Although for better physical appeal the discussion is centered around the sodium-lead system, however, the succeeding treatment of this physical problem is sufficiently general to have applicability to a wide variety of heat and mass diffusion problems with similar characteristics. 
The physical coordinate $x$ is placed at the interface betineen lead and sorium as shown in Fig. 1. This figure also shows schematically temperature and concentration distributions following contact at $x=0$. The melt interface between solid and molten lead is located at $x=\delta(t)$. The equations governing one-dimensional heat and mass diffusion and the motion of molten interface are given as

$$
\begin{aligned}
& \frac{\partial T}{\partial t}=\alpha \frac{\partial^{2} T}{\partial x^{2}} \\
& \frac{\partial C}{\partial t}=0 \frac{\partial}{\partial x}\left(\rho \frac{\partial C}{\partial x}\right) \\
& \rho \Delta H \frac{d \delta}{d t}=\left.K_{S} \frac{\partial T}{\partial x}\right|_{\delta^{+}}-\left.K_{M} \frac{\partial T}{\partial x}\right|_{\delta^{-}}
\end{aligned}
$$

with boundary conditions as

$$
\begin{aligned}
& \left.K_{S} \frac{\partial T}{\partial x}\right|_{L}=0 \\
& r(\delta, t)=T_{M} \\
& T\left(0^{+}, t\right)=T\left(0^{-}, t\right) \\
& \left.K_{M} \frac{\partial T}{\partial x}\right|_{0^{+}}=\left.K_{B} \frac{\partial T}{\partial x}\right|_{0^{-}} \\
& T(-\infty, t)=T_{\infty} \\
& C(\delta, t)=1
\end{aligned}
$$




$$
\begin{aligned}
& c\left(0^{+}, t\right)=c\left(0^{-}, t\right) \\
& \left.D_{M} \frac{\partial C}{\partial x}\right|_{0^{+}}=\left.D_{N} \frac{\partial C}{\partial x}\right|_{0^{-}} \\
& C(-\infty, t)=0
\end{aligned}
$$

and initial conditions as

$$
\begin{aligned}
& T(x, 0)=T_{0}, C(x, 0)=1 \text { for } x>0 \\
& T(x, 0)=T_{\infty}, C(x, 0)=0 \text { for } x<0 \\
& \delta(0)=0
\end{aligned}
$$

Density, $\rho$ of the mixture occurring in equation $(2)$ is related to densities $P_{M}$ and $\rho_{N}$ of pure lead and sodium through

$$
\frac{1}{\rho}=\frac{1-C}{\rho_{N}}+\frac{C}{\rho_{M}}
$$

The above expression clearly shows that if $\rho_{N}$ and $\rho_{M}$ were nearly equal then the mixture density $\rho$ will be nearly constant, however, $\rho_{M}=13 \rho_{N}$, implying that the mixture density $\rho$ cannot be assumed constant. As will be demonstrated subsequently, the usual assumption of constant $\rho$ in solving mass diffusion problem leads to significant errors in the calculation of concentration $C$ from equation (2). Further, we notice that for liquids $a / D \sim$ $10^{4}-10^{5}$, this implies that the boundary layer (or penetration) thickness (which varies as square root of diffusivity and is a measure of the extent of 
diffusion) for mass diffusion, $L_{D}$ is atleast $10^{-2}$ times smaller than the boundary layer thickness for heat diffusion, $L_{\alpha}$. Furthermore, $\delta \sim \sqrt{\alpha_{m}}$ lfor $^{\mathrm{t}}$ an infinite lead $s(a b)$, it then follows that $\left(L_{D} / \delta\right) \sim 10^{-\hat{2}}$. This discussion implies that the thickness of the molten region is always about two order of magnitude greater than mass diffusion boundary layer thickness. Consequently boundary conditions (9) need not be applied at $x=\hat{0}$. As will be seen subsequently, the penetration thickness for mass diffusion is on the order of millimeters over the time span of interest. Thus, for all practical purposes boundary conditions (9) can be modified to read as

$$
C(\infty, t)=1
$$

It follows from the previous discussion that field width of interest for the calculation of mass concentration is considerably smaller than that for the heat diffusion. Furthermore boundary conditions (16b) and (12) imply that for all practical purposes both molten lead and sodium can be considered to be a media .. infinite thicknesses for the purpose of calculating mass concentration. In writing equations (1) and (2) we have implicitly assumed that properties are constant i.e. independent of temperature or concentration field. As will be seen subsequently, the penetration thickness of the concentration both in lead and sodium is extremely small compared to the field width for heat diffusion, thus for all practical purposes mass diffusion is confined in the neighborhood of interface between sodium and lead. It then follows that the properties of the two media for heat diffusion are independent of concentration over almost all the field width. Simflarily it follows diffusion coefficients for these media are also independent of temperature fields. It also follows that the mesh distribution in physical 
coordinates is not suitable for the calculation of concentration as it must be adaptive to account for extreme narrowness of the field width for the concentration. In other words, we must stretch the field width by using a coordinate transformations of the form:

Lead: $\quad n_{C}=1+\exp \left(-\lambda_{M} \times / L\right)$

Sodium: $\quad n_{C}=3-\exp \left(\lambda_{N} \times / L\right)$

where $\lambda_{M} \gg 1$ and $\lambda_{N} \gg 1$ are the stretching parameters. These parameters allow us to stretch the widths to any lesired degree. Furthermore, meshes with these transformations are much more closely spaced in the neighborhood of interface where the variation of concentration is the sharpest. Clearly these transformations transform an infinite domain in physical coordinates into a finite domain in the transformed coordinates.

In order to track the moving melt interface, it is convenient to introduce the following coordinate transformations:

Solid lead (region \#1):

for a slab of finite thickness,

$n=\frac{1-x / L}{1-\Delta}, \Delta=\delta / L$

for a slab of infinite thickness,

$\eta=\exp \left[-B_{S}\left(\frac{x}{[}-\Delta\right)\right]$ 
Molten lead (region \#2):

$$
n=2-\frac{x / L}{\Delta}
$$

Sodium coolant

$$
n=3-\exp \left(\beta_{N} \times / L\right)
$$

The transformaticin (18) allows the transformed coordinate $n$ to vary from 0 to 1 in the solid lead region both for finite and infinite slabs. The transformation (19) for molten lead allows $n$ to vary between 1 and 2 . The transformation (20) for sodium allows $n$ to vary between 2 and 3 . The boundaries of these coordinates are shown in Fig. 1. In these transformed coordinates, the melt interface always lies at $\eta=1$ and consequently appears immobilized in these coordinates. We may note that transformations (17) for the concentration also vary from 1 to 2 in molten lead region and from 2 to 3 in sodium. By maintaining these boundary values of the transformed coordinates, we can use the same mesh distributions for concentration in transformed coordinates as used for heat diffusion in molten lead and sodium, respectively. This in turn will allow us to use the same set of approximating functions for both heat and mass diffusion.

Using transformations (17) through (20) in equations (1) through (12) and (16b) gives: 
Solid lead:

finite siab,

$$
\begin{aligned}
& \frac{\partial \phi}{\partial \tau}=-\frac{\dot{\Delta}}{1-\Delta} n \frac{\partial \phi}{\partial \eta}+\frac{\left(\alpha_{S} / \alpha_{M}\right)}{(1-\Delta)^{2}} \frac{\partial^{2} \phi}{\partial \eta^{2}} \\
& \left.\frac{\partial \phi}{\partial \eta}\right|_{n=0}=0 \\
& \phi(1, \tau)=1
\end{aligned}
$$

infinite slab,

$$
\begin{aligned}
& \frac{\partial \phi}{\partial \tau}=-\beta_{S} \eta \dot{\Delta} \frac{\partial \phi}{\partial \eta}+\left(\alpha_{S} / \alpha_{M}\right) \beta_{S}^{2} \eta\left(\frac{\partial \phi}{\partial \eta}+\eta \frac{\partial^{2} \phi}{\partial \eta^{2}}\right) \\
& \phi(0, \tau)=\phi_{0} \\
& \phi(1, \tau)=1
\end{aligned}
$$

Molten lead:

$$
\begin{aligned}
& \frac{\partial \Psi}{\partial \tau}=-(2-n) \frac{\dot{\Delta}}{\Delta} \frac{\partial \Psi}{\partial \eta}+\frac{1}{\Delta^{2}} \frac{\partial^{2} \Psi}{\partial \eta^{2}} \\
& \psi(1, \tau)=1 \\
& \Psi\left(2^{-}, \tau\right)=\theta\left(2^{+}, \tau\right) \\
& \frac{\partial \omega}{\partial \tau}=\lambda_{M}^{2}(n-1)\left(\frac{D_{M}}{\alpha_{M}}\right)\left[Y_{\rho}(n-1)\left(\frac{\partial \omega}{\partial \eta}\right)^{2}+\frac{\partial \omega}{\partial \eta}+(n-1) \frac{\partial^{2} \omega}{\partial n^{2}}\right]
\end{aligned}
$$




$$
\begin{aligned}
& \omega(1, \tau)=1 \\
& \omega\left(2^{-}, \tau\right)=C\left(2^{+}, \tau\right)
\end{aligned}
$$

Sodium:

$$
\begin{aligned}
& \frac{\partial \theta}{\partial \tau}=\beta_{N}^{2}(\eta-3)\left(\frac{\alpha_{N}}{\alpha_{M}}\right)\left[\frac{\partial \theta}{\partial \eta}+(\eta-3) \frac{\partial^{2} \theta}{\partial \eta^{2}}\right] \\
& \left.\frac{K_{M}}{\Delta} \frac{\partial \psi}{\partial \eta}\right|_{n=2^{-}}=\left.K_{N} \beta_{N} \frac{\partial \theta}{\partial \eta}\right|_{n=2^{+}} . \\
& \theta(3, \tau)=0 \\
& \frac{\partial C}{\partial \tau}=\lambda_{N}^{2}(n-3)\left(\frac{D_{N}}{\alpha_{M}}\left[\gamma \rho(n-3)\left(\frac{\partial C}{\partial \eta}\right)^{2}+(\eta-3) \frac{\partial^{2} C}{\partial \eta^{2}}\right]\right. \\
& \left.D_{M}{ }^{\lambda} \frac{\partial \omega}{\partial \eta}\right|_{n=2^{-}}=\left.D_{N} \lambda_{N} \frac{\partial C}{\partial \eta}\right|_{n=2^{+}} \\
& C(3, \tau)=0
\end{aligned}
$$

Here, in mass diffusion equations (25a) and (27a) we have omitted the use of subscript $c$ on $n$ with a view to using the mesh distribution both $n$ and $n_{c}$.

Melt interface:

finite lead slab,

$$
\dot{\Delta}=\operatorname{Se}\left[\left.\frac{\left(K_{S} / K_{M}\right)}{1-\Delta} \frac{\partial \phi}{\partial \eta}\right|_{n=1^{-}}-\left.\frac{1}{\Delta} \frac{\partial \psi}{\partial \eta}\right|_{n=1^{+}}\right]
$$


$-11$

infinite lead slab,

$$
\dot{\Delta}=\operatorname{Se}\left[\left.\left(K_{S} / K_{M}\right) B_{S} \frac{\partial \phi}{\partial \eta}\right|_{n=1}--\left.\frac{1}{\Delta} \frac{\partial \Psi}{\partial \eta}\right|_{n=1}+\right]
$$

COLLOCATION METHOD

We shall seek an approximate solution of equations $(21-28)$ by a collocation method, using cubic Hermite spline basis functions as approximating functions in the spatial variable $n$ for $F(n, \tau)$ (where $F$ denotes a dependent variable). More specifically, let the interval $[p-1, p]$ pertaining to a ph region $(1<p<3)$ be divided by a set of points, called break points as

$$
p-1=n_{1}<n_{2}<\ldots<n_{\ell+1}=p, \quad n_{i}=n_{i}-n_{i-1}
$$

A convenient basis for generating Hermite splines is the set $\left\{V_{i}(n), S_{i}(n)\right\}_{i=1}^{l+1}$ where

$$
v_{i}(n)= \begin{cases}3\left(\frac{n-n_{i-1}}{n_{i}}\right)^{2}-2\left(\frac{n-n_{i-1}}{n_{i}}\right)^{3} & \text { for } n_{i-1}<n<n_{i} \\ \left(1-\frac{n-n_{i}}{n_{i+1}}\right)^{2}\left[1+2\left(\frac{n-n_{i}}{n_{i+1}}\right)\right] & \text { for } n_{i}<n<n_{i+1} \\ \text { e otherwise } & \end{cases}
$$




$$
s_{i}(n)= \begin{cases}n_{1}\left[-\left(\frac{n-n_{i-1}}{n_{i}}\right)^{2}+\left(\frac{n-n_{i-1}}{n_{i}}\right)^{3}\right] & \text { for } n_{1}<n_{i+1}<n_{i} \\ n_{f+1}\left(\frac{n-n_{i}}{n_{i+1}}\right)\left(1-\frac{n-n_{i}}{n_{i+1}}\right)^{2} & \text { for } n_{i}<n<n_{i+1} \\ 0 \text { otherwise } & \end{cases}
$$

- It is assumed that the function $V_{1}(n)$ and $S_{1}(n)$ vanish to the left of $n_{1}$ and functions $V_{\ell+1}(n)$ and $S_{\ell+1}(n)$ vanish to the right of $n_{\ell+1}$. In addition, we note the following properties of the basis functions:

1. Each $V_{i}(n)$ and $S_{j}(n)$ is continuous together with its derivative in the domain $[p-1, p]$ with degree of smoothness, $v=2$.

2. Each $V_{i}$ and $S_{i}$ is a cubic (i.e., order $k=4$ ) polynomial in each subinterval, and they vantsh outside the interval $\left[n_{i-1}, n_{i+1}\right]$.

3. $\begin{array}{lll}v_{i}\left(n_{j}\right)=\delta_{i j} & v_{j}^{\prime}\left(n_{j}\right)=0 & 1<i, j<\ell+1 \\ s_{i}\left(n_{j}\right)=0 & S_{j}^{\prime}\left(n_{j}\right)=\delta_{i j} & \end{array}$

In terms of these basis functions, a dependent variable $F(n, t)$ can be approximated as

$$
F(n, \tau)=\sum_{i=1}^{\ell+1}\left[F_{i}(\tau) V_{i}(n)+F_{i}^{\prime}(\tau) S_{i}(n)\right]
$$

where the coefficients of expansion $F_{j}(\tau), F_{j}(\tau)$, as follows from property 3 of Hermite splines, are respectively the unknown values of the function $F(n, \tau)$ and its spatial derivative at the break point $\eta_{j}$ with $1<i<\ell+1$; 
i.e., $F_{j}(\tau)=F\left(n_{j}, \tau\right), F_{j}^{\prime}(\tau)=F^{\prime}\left(n_{j}, \tau\right)$. . In view of property 2 of these splines, expansion (30a) becomes

$$
\begin{aligned}
F(n, \tau)= & \sum_{i=j}^{j+1}\left[F_{i}(\tau) V_{j}(n)+F_{i}^{\prime}(\tau) S_{i}(n)\right] \text { for } n \varepsilon\left(n_{j}, n_{j+1}\right) \\
& \text { with } 1<j<\ell
\end{aligned}
$$

The use of expansion (30) in equations (21 - 28) gives, respectively,

$$
\begin{aligned}
& \text { for finite slab, } \\
& \sum_{i=j}^{j+1}\left[\dot{\phi}_{i} v_{i}\left(n^{1}\right)+\dot{\phi}_{i}^{\prime} s_{i}\left(n^{1}\right)\right]=-\frac{\dot{\Delta}}{1-\Delta} n^{1} \phi^{\prime}\left(n^{1}\right)+\frac{1}{(1-\Delta)^{2}} \phi^{\prime \prime}\left(n^{1}\right) \\
& \dot{\phi}_{1}^{\prime}=0 \\
& \dot{\phi}_{\ell+1}=0
\end{aligned}
$$

for infinite slab,

$$
\begin{aligned}
& \sum_{i=j}^{j+1}\left[\dot{\phi}_{i} v_{i}\left(n^{1}\right)+\dot{\phi}_{i}^{\prime} s_{i}\left(n^{1}\right)\right]=-\beta_{S^{n}} n^{\dot{\Delta}} \phi^{\prime}\left(n^{1}\right) \\
& \quad+\beta_{S}^{2} n^{1}\left[\phi^{\prime}\left(n^{1}\right)+n \phi^{\prime \prime}\left(n^{1}\right)\right] \\
& \dot{\phi}_{1}=0 \\
& \dot{\phi}_{\ell+1}=0
\end{aligned}
$$


$-14-$

$$
\begin{aligned}
& \sum_{i=j}^{j+1}\left[\dot{\Psi}_{i} v_{i}\left(n^{2}\right)+\dot{\psi}_{i}^{\prime} S_{f}\left(n^{2}\right)\right]=-\left(2-n^{2}\right) \frac{\dot{\Delta}}{\Delta} \Psi^{\prime}\left(n^{2}\right) \\
& +\left(\frac{a_{M} / a_{S}}{\Delta^{2}}\right) \Psi^{\prime \prime}\left(n^{2}\right)
\end{aligned}
$$$$
\dot{\Psi}_{1}=0
$$

$$
\dot{\Psi}_{\ell+1}-\dot{\theta}_{\ell+1}=0
$$

$$
\begin{aligned}
& \sum_{i=j}^{j+1}\left[\dot{\omega}_{i} v_{f}\left(n^{2}\right)+\dot{w}_{i}^{\prime} S_{f}\left(n^{2}\right)\right]=\lambda_{M}^{2}\left(n^{2}-1\right)\left(\frac{D_{M}}{\alpha_{S}}\right)\left[\gamma \rho\left(n^{2}-1\right)\left[\omega^{\prime}\left(n^{2}\right)\right]^{2}\right. \\
& \left.+\omega^{\prime}\left(n^{2}\right)+\left(n^{2}-1\right) \omega^{\prime \prime}\left(n^{2}\right)\right\}
\end{aligned}
$$

$$
\dot{\omega}_{1}=0
$$

$\dot{w}_{\ell+1}-c_{1}=0$

(34c)

$$
\sum_{i=j}^{j+1}\left[\dot{\theta}_{i} v_{i}\left(n^{3}\right)+\theta_{i} s_{i}\left(n^{3}\right)\right]=\beta_{N}^{2}\left(n^{3}-3\right)\left(\frac{a_{N}}{a_{S}}\right)\left[\theta^{\prime}\left(n^{3}\right)+\left(n^{3}-3\right) \theta^{\prime \prime}\left(n^{3}\right)\right]
$$

(35a)

$$
\begin{aligned}
& \dot{\Psi}_{\ell+1}^{\prime}-\left(K_{N} / K_{M}\right) \Delta \beta_{N} \dot{\theta}_{1}^{\prime}=\left(K_{N} / K_{M}\right) B_{N} \theta_{1}^{\prime} \dot{\Delta} \\
& \dot{\theta}_{\ell+1}=0 \\
& \sum_{i=j}^{j+1}\left[c_{i} V_{i}\left(n^{3}\right)+c_{i}^{\prime} s_{i}\left(n^{3}\right)\right]=\lambda_{N}^{2}\left(n^{3}-3 i\left(\frac{D_{N}}{\alpha_{S}}\right)\left[r_{\rho}\left(n^{3}-3\right)\left[C^{\prime}\left(n^{3}\right)\right]^{2}\right.\right. \\
& \left.+C^{\prime}\left(n^{3}\right)+\left(n^{3}-3\right) C^{: 1}\left(n^{3}\right)\right\}
\end{aligned}
$$




$$
\begin{aligned}
& \dot{\omega}_{\ell+1}^{\prime}-\left(\frac{D_{N}}{D_{M}}\right)\left(\frac{\lambda_{N}}{\lambda_{M}}\right) c_{1}^{\prime}=0 \\
& \varepsilon_{\ell+1}=0
\end{aligned}
$$

finite lead slab,

$$
\dot{\Delta}=\operatorname{Se}\left[\frac{1}{1-\Delta} \phi_{l+1}^{\prime}-\frac{\left(K_{M} / K_{S}\right)}{\Delta} \Psi_{1}^{\prime}\right]
$$

infinite lead slab,

$$
\dot{\Delta}=\operatorname{Se}\left[\hat{B}_{S} \phi_{l+1}^{\prime}-\frac{\left(K_{N} / K_{S}\right)}{\Delta} \Psi_{1}^{\prime}\right]
$$

Here $n^{p}$ denotes $n$ pertaining to $p$ th region $(1<p<3)$ and

$$
\begin{aligned}
& F^{\prime}\left(n^{p}\right)=\sum_{i=j}^{j+1}\left[F_{i} V_{i}^{\prime}\left(n^{p}\right)+F_{i}^{\prime} s_{j}^{\prime}\left(n^{p}\right)\right] \\
& F^{\prime \prime}\left(n^{p}\right)=\sum_{i=j}^{j+1}\left[F_{i} V_{i}^{\prime \prime}\left(n^{p}\right)+F_{i}^{\prime} S_{j}^{\prime \prime}\left(n^{p}\right)\right]
\end{aligned}
$$

which holds for $n^{p} \varepsilon\left(n_{j}^{p}, n_{j+1}^{p}\right)$ with $1<j<l_{p}$ and $1<p<3$. For later convenience in obtaining a solution, we have taken the time derivative of the boundary condition after using expansion (30) in them.

Each of these equation sets $(31-36)$ contain $N_{p}=2\left(e_{p}+1\right)$ with $1<p<3$ unknown coefficients of expansion. The two boundary conditions associated with each set naturally allow us to determine two coefficients. The remaining unknown coefficient equal to $2 l_{p}$ in each set are stemined by evaluating or collocating these PDEs at $2 e_{p}$ collocation points in each 
region. According to approximation theory $[3,4]$ optimum collocation points for Hermite splines as the approximating functions, are the Gauss-Legendre quadrature points of order 2 for each interval:

$$
\sigma_{j, q}^{p}=\frac{1}{2}\left(n_{j}^{p}+n_{j+1}^{p}\right)+(-1)^{q} \frac{n_{j+1}^{p}-n_{j}^{p}}{2 \sqrt{3}} \quad 1<j<\ell_{p}, 1<q<2
$$

The evaluation of each of the PDEs in the set $(31-36)$ at the above mentioned collocation poirits leads to a set of ODEs. These equations when combined with their boundary conditions and equation (37) for melt interface give the following set of ODEs written compactly as

$$
A(Y, \tau) \hat{Y}=G(Y, \tau)
$$

where $Y$ is an $5 N_{p}+1(1<p<3)$ dimensional vector of $5 N_{p}$ unknown coefficients and the position of melt interface. By virtue of the local or piecewise basis of the approximating functions, the coefficient matrix $A(Y, \tau)$ is banded matrix of bandwidth equal to $2(2 k-1)+1$.

\section{COMPUTATIONAL DETAILS}

The solution of equation (40) is obtained by the standard library routine LSODI [5]. It solves the initial value problem with banded coefficient matrix by using Gear's so-called backward multistep differentiation formulas suitable for stiff system. The size of time step is controlled automatically by specified error tolerance for the integrator.

For the system of equations $(31-37)$ it is clear that at time $\tau=0$, the system is singular, consequently we must provide a starting solution which is approximately valid in the neighborhood of $\tau=0$. One $c$ an readily observe that finite lead slab behaves like an infinite slab during the short time 
after it is brought into contact with the sodium pool. Consequently one can use an analytical solution avaliable [6] for describing temperature profiles and the position of the melt interface subsequent to contact between two infinite slabs initially at uniform but different temperatures. This solution is given as

$$
\begin{aligned}
& \phi(x, t)=\phi_{0}+\frac{1-\phi_{0}}{\operatorname{erfc}\left(\mu \sqrt{\alpha_{M} / \alpha_{S}}\right)} \operatorname{erfc}\left(\frac{x}{2 \sqrt{\alpha_{S} t}}\right) \\
& \psi=\frac{K_{M} \sqrt{a_{N}}+K_{N} \sqrt{\alpha_{M}} \operatorname{erf}\left(\frac{x}{2 \sqrt{\alpha_{M} t^{t}}}\right)}{K_{M} \sqrt{\alpha_{N}}+K_{N} \sqrt{\alpha_{M}} \operatorname{erf}(\mu i} \\
& \theta=\frac{K_{M} \sqrt{\alpha_{N}}}{K_{M} \sqrt{\alpha_{N}}+K_{N} \sqrt{\alpha_{M}} \operatorname{erf}(\mu)} \operatorname{erfc}\left(-\frac{x}{2 \sqrt{\alpha_{N} t}}\right) \\
& \delta_{\infty}=2 \mu \sqrt{\alpha_{M} t} \\
& \frac{K_{N} \sqrt{\alpha_{M}} e^{-\mu}}{K_{M} \sqrt{\alpha_{N}}+K_{N} \sqrt{\alpha_{M}} \operatorname{erf}(\mu)}-\frac{K_{S} \sqrt{\alpha_{M}} \phi_{0} \exp \left[-\left(\alpha_{M} / \alpha_{S}\right)^{2}\right]}{K_{M} \sqrt{\alpha_{S}} \operatorname{erfc}\left(\mu \sqrt{\alpha_{M} / \alpha_{S}}\right)}=\frac{\mu \sqrt{\pi}}{S e}
\end{aligned}
$$

The starting solution for concentration is provided by analytical solution obtained by assuming $\gamma=0$ i.e. constant mixture density and is given as

$$
\begin{aligned}
& \omega=1-\frac{\sqrt{\sigma_{N}}}{\sqrt{\sigma_{N}}+\sqrt{\sigma_{M}}} \operatorname{erfc}\left(\frac{x}{2 \sqrt{\sigma_{M} t}}\right) \\
& c=\frac{\sqrt{\sigma_{M}}}{\sqrt{\sigma_{N}}+\sqrt{\sigma_{M}}} \operatorname{erfc}\left(-\frac{x}{2 \sqrt{D_{N} t}}\right)
\end{aligned}
$$

The above solution is significantly different (as will be demonstrated subsequentiy) from the solution for the case of variable mixture density with 
widely different densities. However, the numerical solution of equations (25) and (27) converges asymptotically to the "true" solution which does not depend on the starting solution. This is an expected cehavior of this class of problems relating to infinite media.

\section{RESULTS}

Assuming the lead slab to consist of infinite thickness Fig. 2 shows a comparison between the analytical and calculated solution obtained by collocation method for temperature profile. The two solutions agree extremely well with no discernable difference between them. Figure 3 shows the position of the melt interface as a function of time for $L+\infty, L=0.2 \mathrm{~m}$ and $L=0.1 \mathrm{~m}$. The comparison of the calculated solution for case of $L+\infty$ agree again with the analytical solution with no discernable difference between the two. For cases $L=0.2 \mathrm{~m}$ and $L=0.1 \mathrm{~m}$, the calculated solutions as expected agree well for short times with analytical solution for the case of $L+\infty$. After the temperature boundary layer penetrates the thickness of the lead slab, i.e. as the whole thickness of the solid lead begins to heat up, melt interfaces for these two cases begin to move significantly faster than the melt interface for the case $L \rightarrow \infty$. As expected for the melt rate for $L=0.2 \mathrm{~m}$ is slower than that for $L=0.1 \mathrm{~m}$.

Figure 4 shows a plot of concentration profiles as a function of position $b$ th for the cases of constant and variable mixture densities. This figure clearly shows that a signiflcant difference exists between the two profiles, implying that it would not be valid without incurring slgnificant error to use constant density approximation for $\mathrm{Pb}-\mathrm{Na}$ liquid-liquid system. In this figure we have also compared analytical solution with the calculated solution for the case of constant density approximation. These solutions are in excellent 
agreement. One may, however, note that the penetration thickness for both the variable and the constant cases are nearly identical. By comparison of fig. 2 with Fig. 4, one can conclude very readily that penetration thickness for interdiffusing liquids is extremely small as compared to that for temperature. It is thus clear that the computations for mass diffusion in liquids requires the use of very special adaptive coordinates which must cause significant stretching of the physical coordinates.

Figure 5 display the effect of the variation of $Y$ on concentration profiles. As expected with decrease in the value of $Y$, the divergence between the concentration profiles decreases very rapidly. For $\gamma<0.5 \times 10^{-3} \mathrm{~m}^{3} / \mathrm{kg}$, this figure shows that the constant density approximation is a reasonably vaiid approximation for the calculation of concentration profiles for diffusion in liquids.

We have also studied the effect of a difference in diffusion coefficients for the two interdiffusing liquids. This difference can arise if the diffusion coefficients were allowed to $d$ epend on concentration and temperature. Figure 6 shows plots of concentration profiles for various values of diffusion coefficients for sodium. The value for the lead is kept fixed at its calculated nominal value. Is expected, penetration thickness in sodium increases with increasing diffusion coefficient, however, these increases are still very modest, even when the diffusion coefficient in sodium is increased by 10 fold. It would then appear that liquid-liquid diffusion cannot be relied upon to cause any significant mixing between lead and sodium. The two liquid metals will stay separated unless some convective mechanism for mass transport is brought into play. It is thus clear before any significant mixing takes place, lead will sink to the bottom of the steel shot bed upon complete meiting, and will not participate to any significant 
extent in removing heat by natural convection from the fuel debris lying on the top of the steel shot bed.

\section{CONCLUSIONS}

Simultaneous calculations of mass and heat transfer in a liquid-liquid system cannot be carried out in physical coordinates without using a number of meshes that far exceeds the number that is theoretically necessary for normal engineering accuracy. The introduction of the adaptive coordinates which cause considerable stretching of the physical. coordinates, allows the calculation of mass diffusion simultaneous with the heat diffusion and phase change. A comparison of the calculated results with analytical solutions for heat diffusion and melt interface position for the case of infinite lead slab show an excellent agreement. The calculation of the melt interface show that rates of melting increase significantly with decreasing thickness of lead. For the case of constant mixture density, the analytical solution for concentration profile agrees extremely well with the calculated results.

The calculations for concentration profiles show that constant density approximation is not a valid approximation for liquid-liquid diffusion in a binary system such as lead and sodium which have exceedingly different densities. Liquid-liquid mass diffusion is an extremely poor mechanism for causing mixing of one liquid into another and therefore cannot be relied upon without some convective mechanism to achieve any degree of mixing between lead and sodium in PAHR applications.

\section{ACKNOWLEOGEMENTS}

The authors greatly apprectate the skill offered by James Bingle in preparing computer plots of the computational results. This work was performed under the auspices of the U. S. Department of Energy. 


\section{REFERENCES}

1. T. C. Chawla, G. Leaf, and W. Chen, "A Collocation Method Using B-Splines for One-dimensional Heat or Mass Transfer-Controlled Moving Boundary Problems," Nucl. Eng. Des.. 35, 163-180 (1975).

2. T. C. Chawla, G. Leaf, and W. J. Minkowycz, "A Collocation Method for Convection Dominated Flows," Accepted for publication in Int. J. for Numerical Methods in Fluids.

3. J. Douglas and T. Dupont, "A Finite Element Collocation Method for Quasilinear Parabolic Equation," Math. Comput., 27, 17-18 (1973).

4. C. DeBoor and B. Swartz, "Collocation at Gaussian Points," SIAM (Soc. Ind. App i. Math), J. Numer. Anal., 10, 582-606 (1973).

5. A. C. Hindmarsh, "Two New Initial Value Ordinary Differential Equations Solvers," ACM-SIGNUM Newsletter, 15, 10-11 (1980).

6. H. S. Cárslaw and J. C. Jaeger, Conduction of Heat in Solids, Second Edition Oxford University Press, London, 1959. 


\section{NOMENCLATURE}

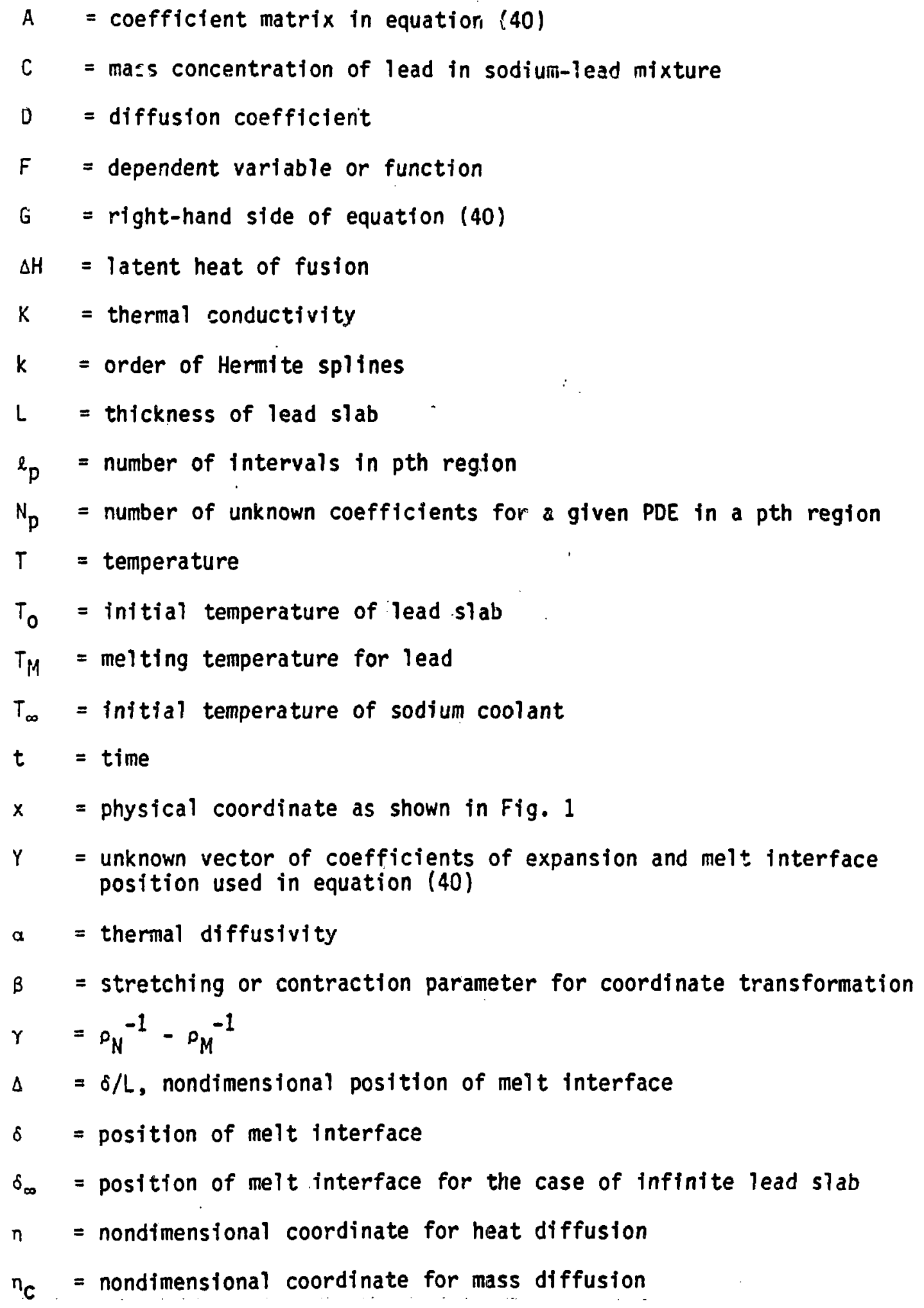


$\theta \quad=\left(T-T_{\infty}\right) /\left(T_{M}-T_{\infty}\right)$, nondimensional temperature for liquid sodium

$u \quad=$ proportionality parameter for melt interface position

D = density of mixture of lead and sodium

$\rho_{M}=$ density of pure molten lead

$\rho_{N}=$ density of pure sodium coolant

$\sigma_{j, q}^{p}=$ position of Gauss-Legendre quadrature point of order $q$ used as a collocation point in $j$ th interval and pth region

$\tau \quad=t_{\alpha} / L$, nondimensional time

$\phi \quad=\left(T-T_{\infty}\right) /\left(T_{M}-T_{\infty}\right)$, nondimensional temperature for solid lead

$\phi_{0}=\left(T_{0}-T_{\infty}\right) /\left(T_{M}-T_{\infty}\right)$, initial nondimensional temperature for solid lead

$\Psi=\left(T-T_{\infty}\right) /\left(T_{M}-T_{\infty}\right)$, nondimensional temperature for molten lead

$\omega=$ mass concentration for lead in lead-sodium mixture in the molten lead region (see Fig. 1)

\section{Subscripts}

$M=$ molten lead

$\mathrm{N} \quad=$ sodium coolant

$p=$ pth region, $1<p<3$

$S=$ solid lead 

(1)
(2)
(3)
REGION NUMBER

SOLIO

LEAD
MOLTEN

LEAD
SODIUM

COOLANT

$\eta=0$

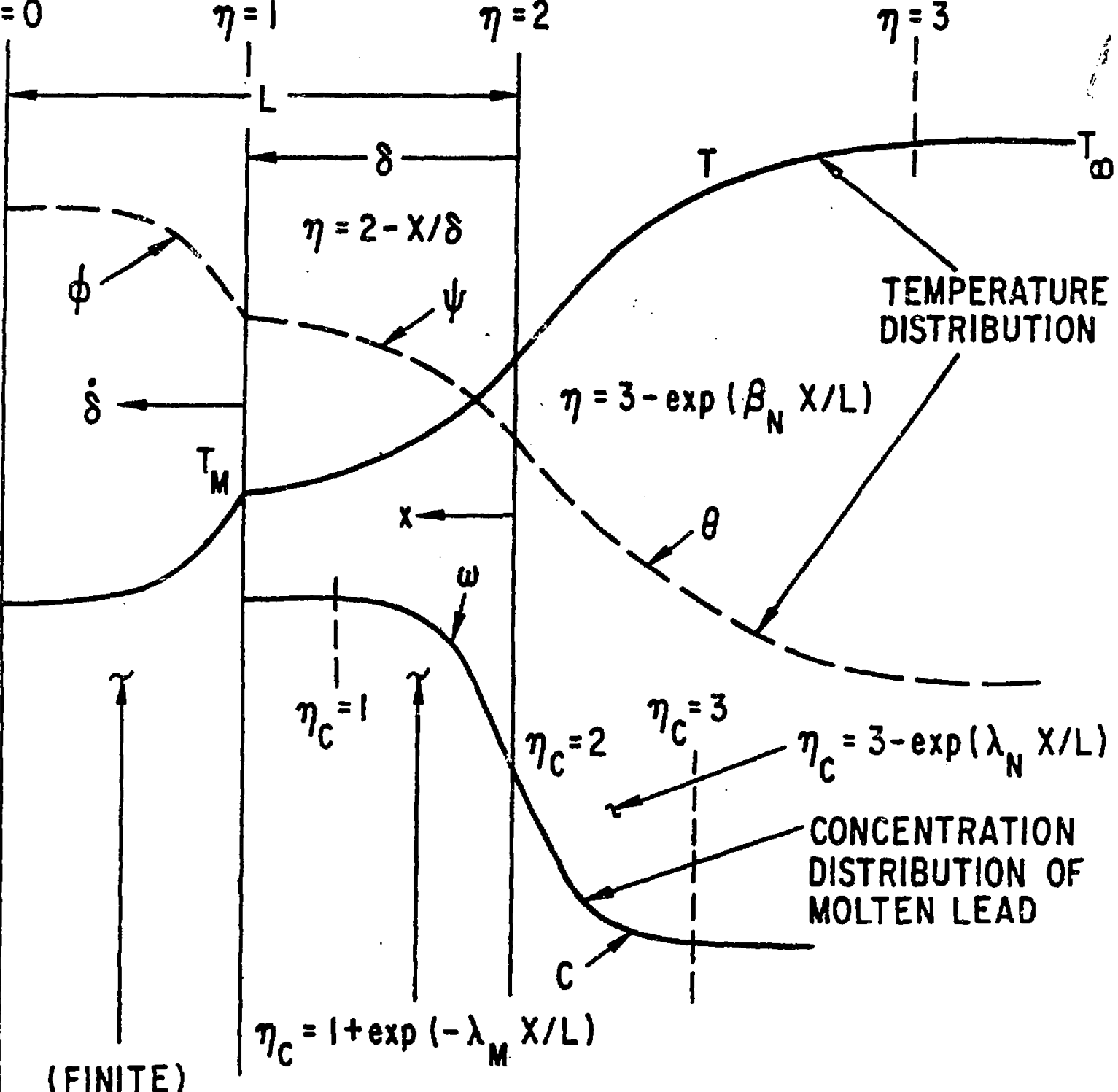

$\eta=(1-X / L) /(1-8 / L)$

(INFINITE)

$\eta=\exp \left[-\beta_{s}(X-\delta) / L\right]$

Fig. 1. Schematic of lead melting and diffusion in sodium following their contact. 


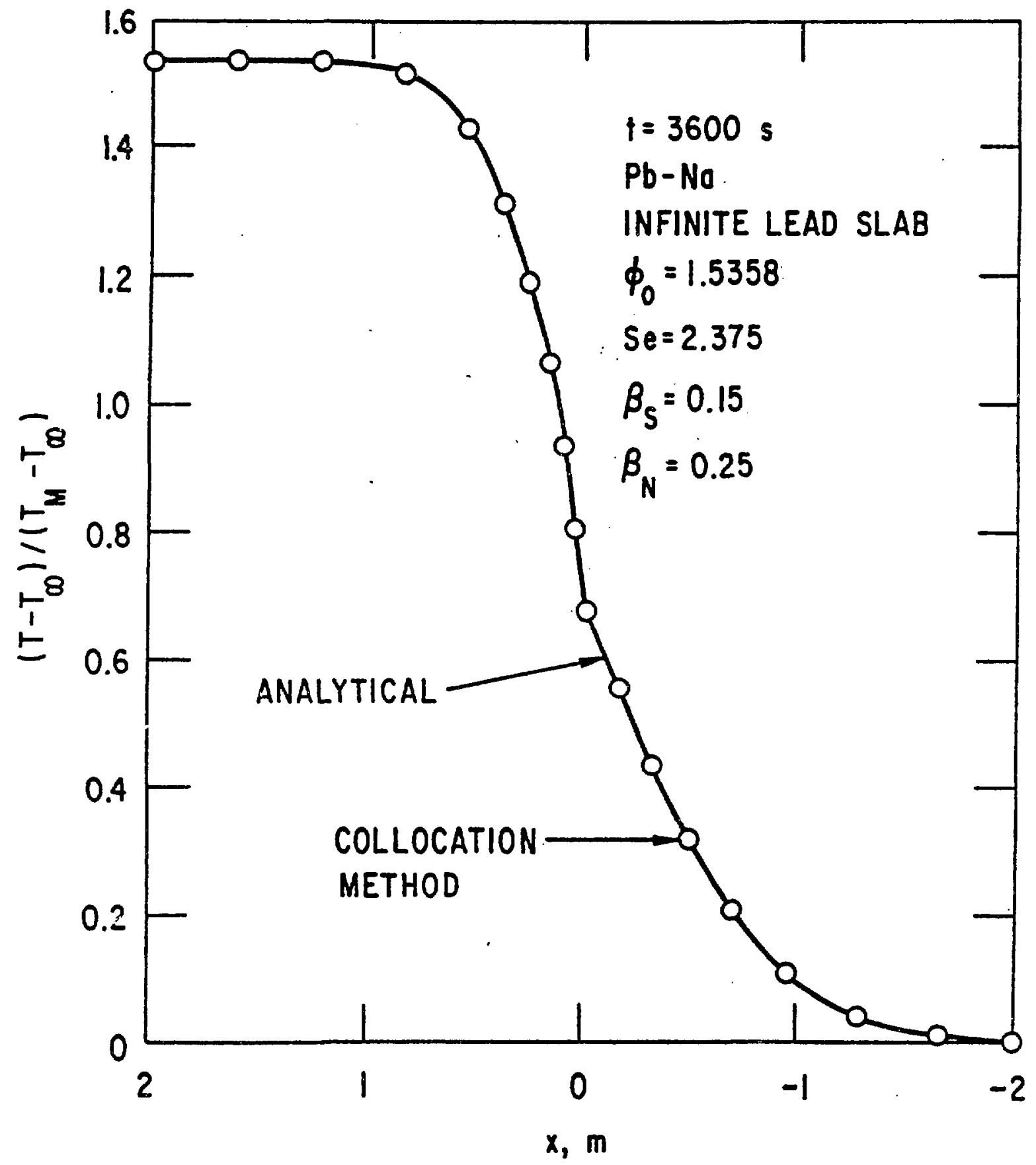

Fig. 2. A comparison of analytical and numerical solutions for teilperature profile for the case of infinite lead slab. 


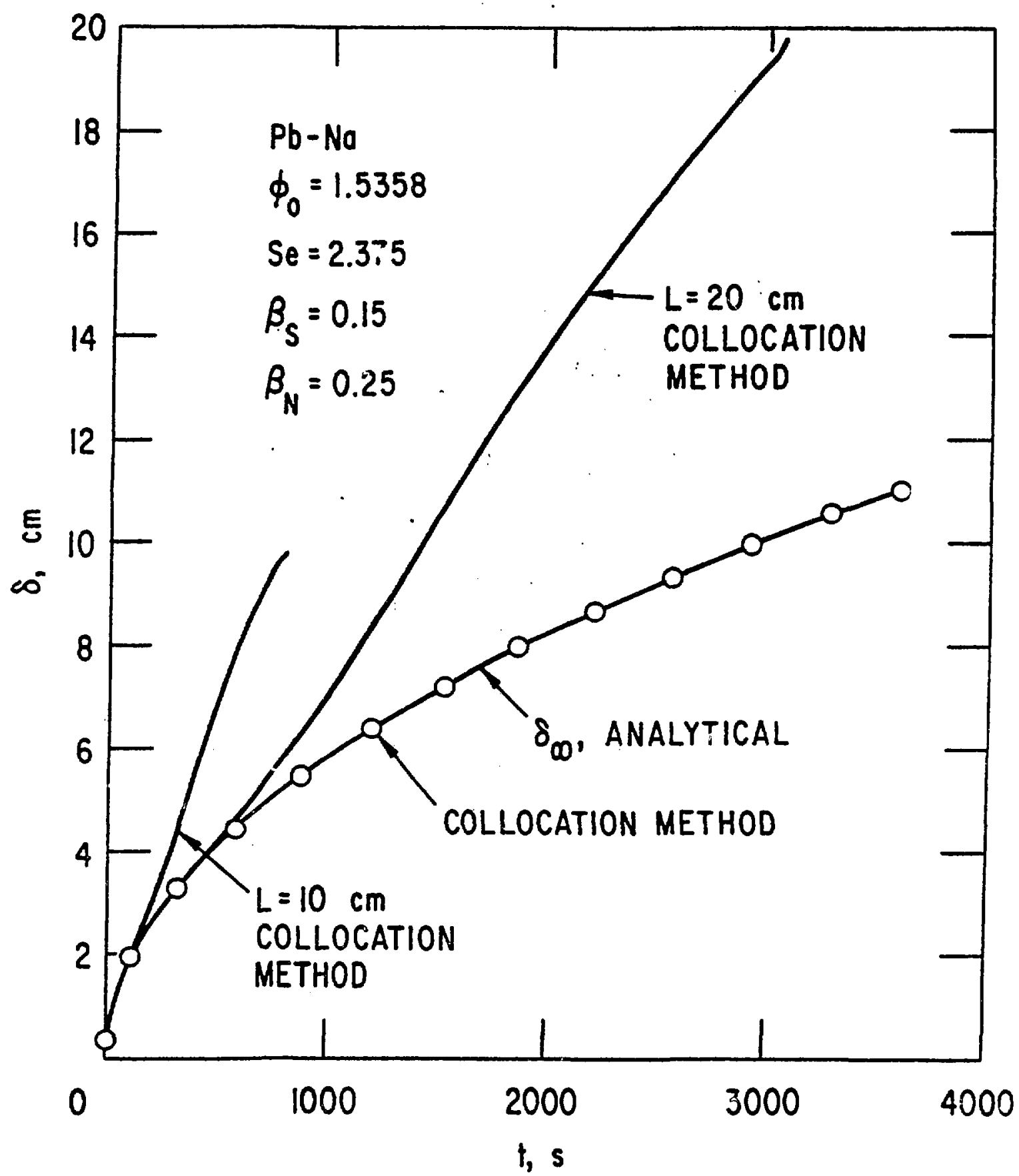

Fig. 3. The position of melt interfaces as a function of time for various thicknesses of the lead slab. 


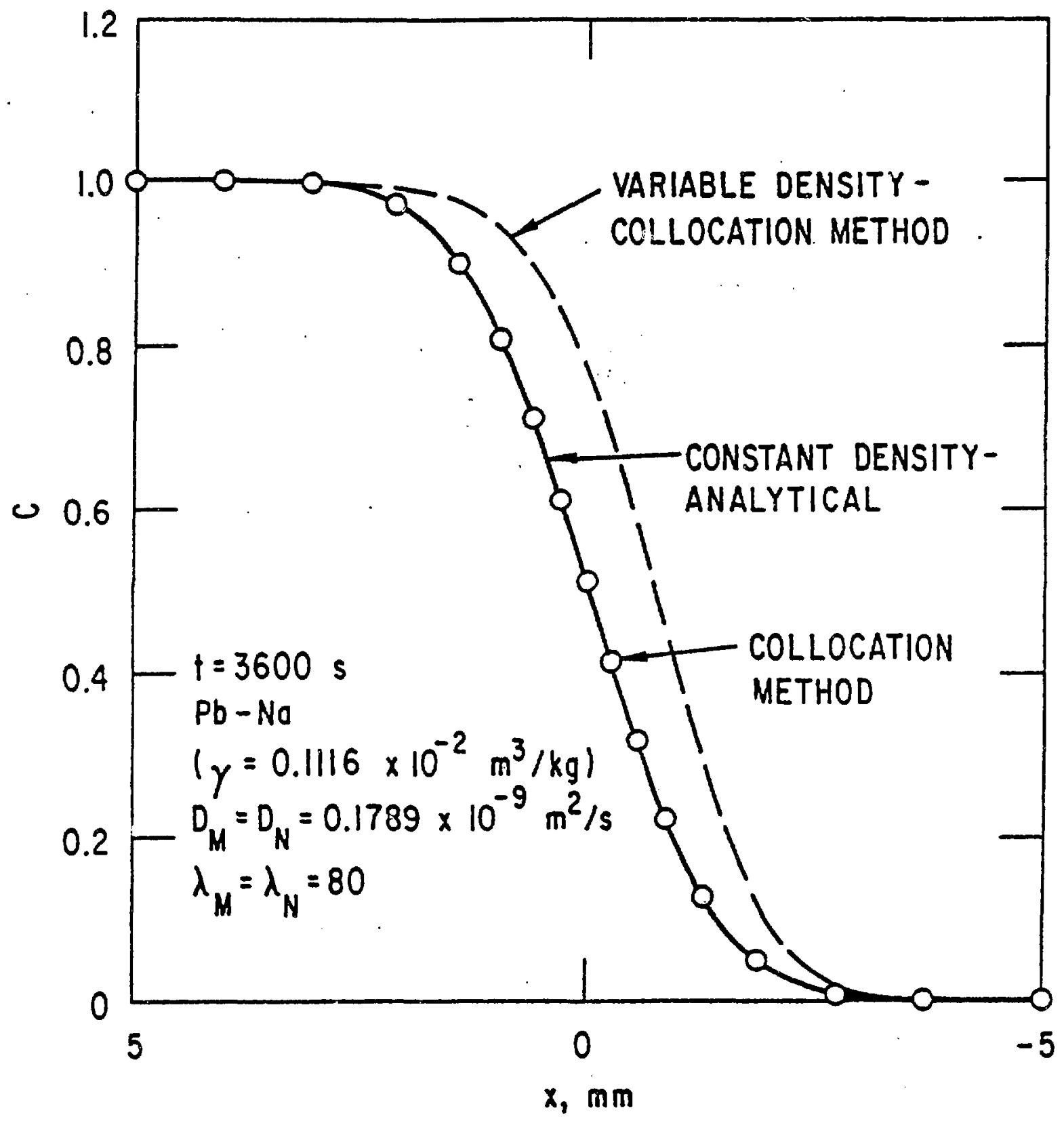

Fig. 4. A comparison of concentration distributions for constant and variable density cases. 


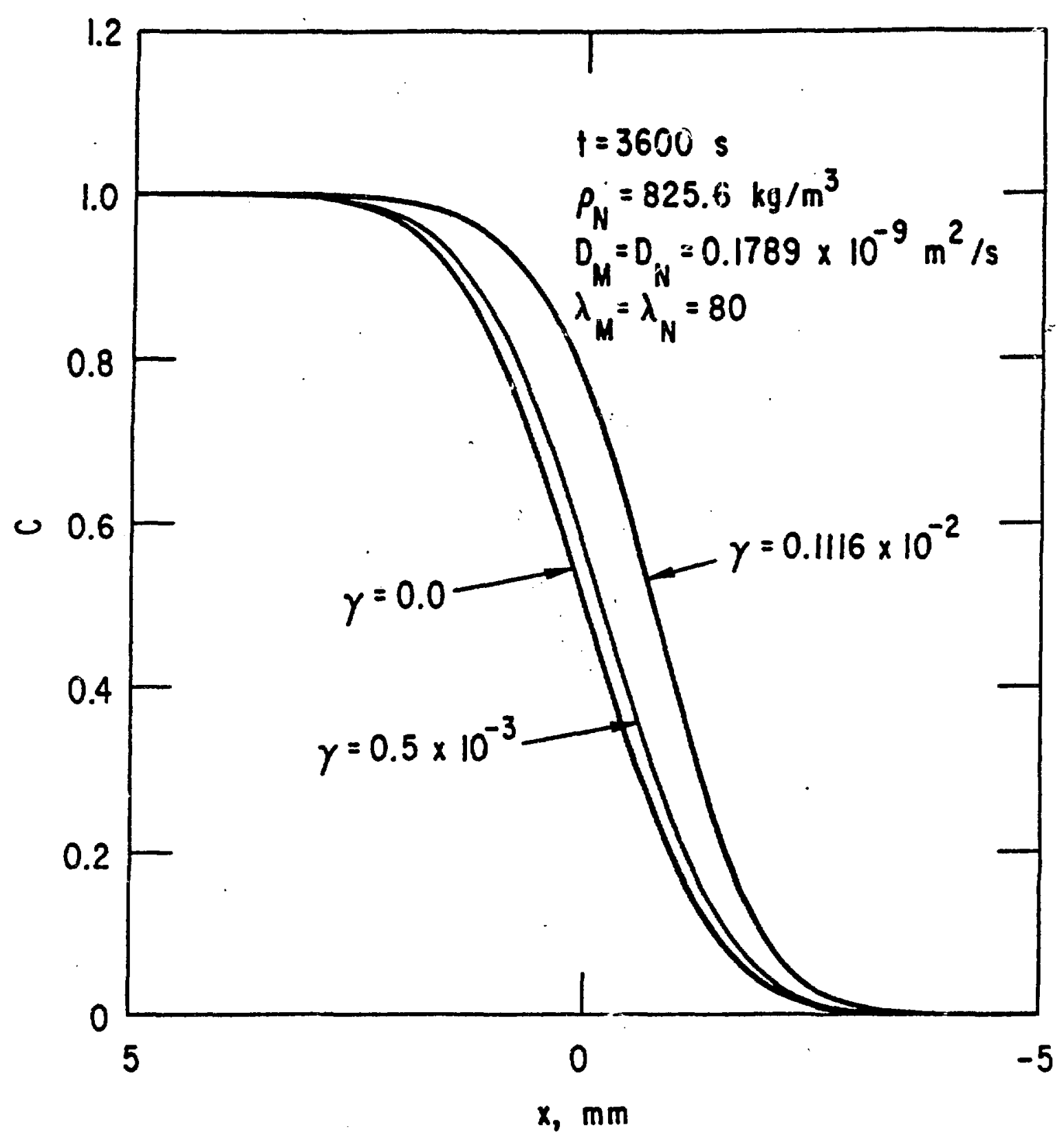

Fig. 5. The effect of parameter $\gamma$ on the concentration profiles. 


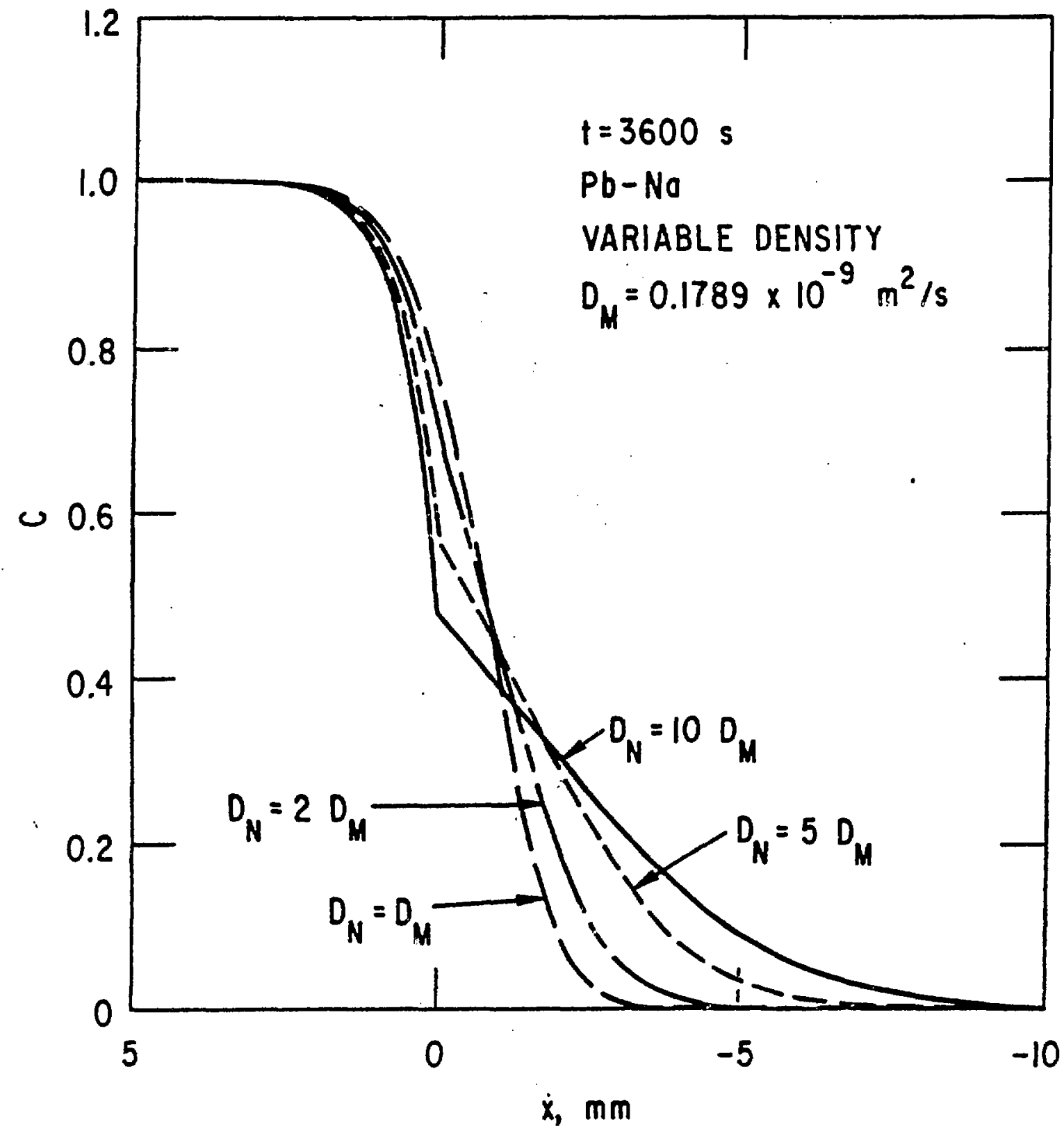

Fig. 6 . The effect of varying diffusion coefficient in sodium on concentration profiles. 